\title{
Potential of Müller glia for retina neuroprotection
}

Karen Eastlake, Joshua Luis, G Astrid Limb

UCL Institute of Ophthalmology and NIHR Biomedical Research Centre at Moorfields Eye Hospital, London, UK

Corresponding author:

G Astrid Limb, PhD, FRCPath

UCL Institute of Ophthalmology

11-43 Bath Street, London EC1V 9EL

Phone: 020 7608-6974

Email: g.limb@ucl.ac.uk 


\begin{abstract}
Müller glia constitute the main glial cells of the retina. They are spatially distributed along this tissue, facilitating their close membrane interactions with all retinal neurons. Müller glia are characterized by their active metabolic functions, which are neuroprotective in nature. Although they can become reactive under pathological conditions, leading to their production of inflammatory and neurotoxic factors, their main metabolic functions confer neuroprotection to the retina, resulting in the promotion of neural cell repair and survival. In addition to their protective metabolic features, Müller glia release several neurotrophic factors and antioxidants into the retinal microenvironment, which are taken up by retinal neurons for their survival. This review summarizes the Müller glial neuroprotective mechanisms and describes advances made on the clinical application of these factors for treatment of retinal degenerative diseases. It also discusses prospects for the use of these cells as a vehicle to deliver neuroprotective factors into the retina.
\end{abstract}

Keywords: Müller glia, retina, neuroprotection, neurotrophins, antioxidants, retinal degeneration 


\section{Müller glia as the backbone of the neural retina}

Müller cells constitute the main macroglia of the neural retina. They span radially across the whole width of the retina, forming a scaffold that provides structural orientation and metabolic support to all neurons $(1,2)$. Müller glia provide tensile strength and resistance to mechanical stress of the retina (3), and this is demonstrated by observations during zebrafish development, in which ablation of these cells leads to retinoschisis and softening of the retina (3), as well as retinal disorganisation, thinning of the photoreceptor layers and increased volume of retinal blood vessels (4). Their ablation also leads to photoreceptor apoptosis, blood-retinal barrier breakdown and sub-retinal neovascularisation (5). Müller cells exhibit a radially oriented morphology with their cell bodies residing within the inner nuclear layer, their apical processes extending towards the outer nuclear layer, and their end feet spreading towards the nerve fibre layer of the retina (1) (illustrated in Fig 1). On their apical end, Müller glia microvilli make direct contact with the photoreceptor inner segments, while their end feet processes closely wrap around dendrites and axons of retinal ganglion cells and blood vessels (1). Using membrane-targeted fluorophores to examine fine details of Müller cell anatomy in the mouse retina, it has been shown that that these cells regularly space themselves, with their processes showing little overlap between the cellular domains of other neighbouring Müller cells, which ensures the complete coverage of the retinal space (6). The number and density of Müller glia vary between species and their location within the retina (7). For example, in the rabbit retina, Muller glia are most abundant in the central retina where the highest number of neurons is observed. This density however is markedly reduced at the peripheral retina where fewer neurons populate this region (8).

The anatomical distribution of Müller glia allows these cells to perform distinct functions throughout the retina. Their processes nearer the sub-retinal space contain golgi, microtubules and vesicles (9) which are thought to be involved in signalling of glutamate, neurotrophic factors and growth factors (10). At the apical site where Müller cells form junctions with the photoreceptors, they show asymmetrical distribution of actin, myosin, $\alpha$-actinin and vinculin, which are all associated with cytoskeletal transport and possibly aid in their special function on the formation of these junctions (11). In contrast, the inner processes of Müller cells contain smooth endoplasmic reticulum, intermediate filaments (such as vimentin), glycogen, aquaporin-4 and $\mathrm{K}+(9)$. Comparative studies between vascular (eg. Cat, opossum) and avascular retinae (eg. Rabbit, turtle) indicate that vascularity appears to influence the distribution of organelles throughout the Müller cell cytoplasm. In the vascularized retina, mitochondria are mainly localized towards the end feet, whilst few mitochondria are observed in the apical region (9). In the plexiform layers, the lateral processes of Müller cells ensheath neural synapses (6) and in general do not contain any organelles (9). These structures are thought to be mainly involved in homeostasis of the extracellular environment, facilitating ion transport exchanges and neurotransmitter uptake (9).

Müller glia provide retinal neuroprotection by producing neurotrophins, regulating water and ion homeostasis, controlling antioxidative functions and recycling neurotransmitters. In contrast to their beneficial metabolic role within the neural retina, they are also known to mediate pathological processes of retinal wound healing and neovascularization, and massive proliferation of these cells is a key feature of retinal proliferative disorders. This is known as reactive gliosis, which is accompanied by release of vasoactive molecules and pro-inflammatory factors by these cells (12). These functions will be described in more detail below. 


\section{Main metabolic features of Müller glia contribute to neuronal protection}

Müller cells are considered the main regulators of neuronal signalling within the retina, a function that they accomplish through the recycling of neurotransmitters. The main excitatory neurotransmitter in the retina is glutamate, which is released by photoreceptors, bi-polar cells and retinal ganglion cells at their synapses. Although glutamate modulates neuronal excitability and synaptic transmission, excessive glutamate can cause over-excitation of neurons, leading to cell death. To protect neurons, Müller glia takes up excess glutamate from the extracellular environment and converts it into glutamine, a function that is exerted through the expression of the enzyme glutamine synthetase (13). Consequently, the uptake of glutamate by Müller glia is considered to be an important neuroprotective function, as inhibition of glutamate transporter uptake or inhibition of glutamine synthetase in Müller glia can rapidly lead to neuronal toxicity, even at very low concentrations (14). Müller glia are therefore important regulators of the neurotransmitter pool, where bi-polar and retinal ganglion cells rely on the glutamine released from Müller glia to synthesise glutamate (15). It has been suggested that Müller glia contribute to visual resolution signalling in the outer retina, by preventing glutamate from diffusing from the synaptic area (16).

Glutamate transport in the mammalian retina is regulated by several sodium dependent excitatory amino acid transporters: GLAST, GLT-1, EAAC1, EAAT4, and EAAT5, although these are expressed by different retinal cell types $(17,18)$. They mediate the transport of glutamate, l-aspartate and $d$ aspartate. The main glutamate transporter is the L-glutamate-L-aspartate (GLAST/EAAT1)(18), which is located throughout the length of the Müller cell membrane. Knockout of this transporter in the mouse results in reduction of bi-polar and Müller cell functions as assessed by a reduction in the bwave of the electroretinogram. They also show a marked increase in the retinal levels of glutamate caused by the lack of this transporter (19). Furthermore, induced ischaemia in this knock-out model intensifies retinal injury (19), and this is a reflection of the impairment of neuroprotection exerted by these cells.

In addition to its excitatory regulation, Müller glia also maintain the retinal extracellular gammaaminobutyric acid (GABA) levels in the retina. GABA is the main inhibitory neurotransmitter in the CNS, and is used by photoreceptors, horizontal, amacrine, b-polar and ganglion cells in the retina (20). Müller glia contribute to synaptic inhibition by maintaining the extracellular levels of this molecule through their expression of GABA transporters (GAT1-4) on their cell membrane (21). Activation of GABA transporters by extracellular GABA is characterized by membrane depolarization and consequent activation of inwardly directed currents due to $\mathrm{Na}^{+}$transport into the cell. Expression of the GAT subtypes depends on the species, but mammalian Müller cells appear to mainly express GAT1 and GAT3 (22).

Müller glia are also involved in the regulation of glycogen and glucose in the retina. Evidence for the storage (mainly towards their end feet), synthesis and regulation of glycogen, as well as the expression of glycogen phosphorylase, a key enzyme for the degradation of this polysaccharide molecule have been demonstrated in these cells (23). Following uptake from the extracellular environment, glucose undergoes phosphorylation within Müller glia, preventing extracellular diffusion and promoting its storage in the form of glycogen. It has been suggested that levels of glycogen storage by these cells may control the distribution and control of the retinal metabolism in order to meet the different metabolic demands of light and darkness. Their intracellular levels of glycogen appears to relate to vascularization, as species with fewer blood vessels require more glycogen storage (24). During metabolic stress, glycogen degradation within Müller glia provides the retina with essential metabolites such as lactate (24), and evidence has been presented that lactate 
released from Müller glia can be utilised by photoreceptors for their mitochondrial oxidative metabolism (25).

Another important function of Müller glia is the removal of excess water from the inner retina. This function is osmotically combined with the transport of ions. These functions are facilitated by their expression of the water channel protein aquaporin-4 (AQP4) and the inward rectifying $\mathrm{K}^{+}$channel subunit Kir4.1 (26). The removal of extracellular $\mathrm{K}^{+}$ions generated by neural excitation within the retina is undertaken by Müller cells in a process called spatial buffering, which is thought to maintain the neuronal information processing. This type of buffering in the retina is referred to as $\mathrm{K}^{+}$siphoning and relies on the distribution of the Müller cell $\mathrm{K}^{+}$conductance (27). In non-mammalian vertebrates and mammals with avascular retinae, the Kir channels are predominantly localized in the Müller cell end foot, whereas in the vascularized mammalian retinae, Müller cell $\mathrm{K}^{+}$conductance is mainly effected in regions where these cells contact the retinal blood vessels (27).

Other neuroprotective effects of Müller cells include the upregulation of adenosine 5 -triphosphate (ATP)-degrading ectoenzymes, which occurs during gliosis. These enzymes enhance the extracellular availability of adenosine, a neuroprotectant nucleoside, as well as abrogation of the osmotic release of ATP, protecting retinal ganglion cells from apoptosis (28). In addition to the Müller metabolic features described above, which directly or indirectly confer neuroprotection in the retina, these cells also secrete a vast number of factors often in response to injury, that either promote inflammation and gliosis, or protect retinal neurons from injury or death. These include cytokines (29), neurotrophins $(30,31)$ and antioxidants $(32,33)$, which play a diversity of roles in the neural retina during health and disease. The main functions of these molecules will be described in the following sections.

\section{Müller glia are an important source of neurotrophins within the retina}

Neurotrophins are important not only for promotion of neuronal survival during development and after injury, but for regulation of apoptosis of retinal neurons (34). They promote axon growth, dendrite pruning, the patterning of neural innervation and the expression of key proteins that regulate neuronal functions, such as neurotransmitters and ion channels (2). Neurotrophins belong to a family of highly conserved proteins which includes nerve growth factor (NGF), brain derived neurotrophic factor (BDNF), neurotrophin-3 (NT-3) and neurotrophin-4 (NT-4) (35). The regulatory influence of neurotrophins is mediated through two independent classes of receptors: the Trk family of high-affinity tyrosine kinase, which include the TrkA, TrkB, and TrkC receptors, and the low-affinity p75 neurotrophin receptor (p75 NTR) (36). In general, downstream targets of TrK receptor activation result in pro-survival signalling via the ERK/MAPK and PI3K pathways; whereas $p 75^{\mathrm{NTR}}$ activation induces a mixture of pro-apoptotic pathways via JNK and a variety of other pathways including NF-KB (37). A further complexity exists in that whilst $p 75^{\mathrm{NTR}}$ can bind to all neurotrophins, it can also act as a co-receptor for Trk, modulating it into a higher affinity state (38). Although it is generally thought that Trk and p75 receptors trigger opposing signalling systems to promote cell survival and death, it has been proposed that this view may be oversimplified as the complex signalling network of neurotrophins is not completely understood (36).

Neurotrophins are translated as large $(130 \mathrm{kDa})$ pro-polypeptides, known as pro-forms, that undergo post-translational proteolytic cleavage to exert their neuroprotective function (39). Pro-forms have been shown to preferentially activate $\mathrm{p} 75^{\mathrm{NTR}}$ to mediate apoptosis, whilst activation of TrK receptors by mature forms promote survival. This is illustrated by observations with the pro-forms of NGF, BDNF and NT3, which have a high-affinity ligand for $p 75^{\mathrm{NTR}}$. Upon binding to this receptor, these neurotrophins form a heterotrimeric complex that have a marked pro-apoptotic effect in opposition 
to their mature forms (39). NGF, BDNF and NT3 have been shown to be secreted as pro-forms and cleaved extracellularly by proteases into the mature forms $(39,40)$. As part of their neuroprotective function, Müller glia produce selective neurotrophins under different conditions. In response to glutamate Müller glia upregulate their secretion of BDNF, NGF, NT-3, NT-4, and GDNF, suggesting that by releasing neurotrophins, these cells play an important role in preventing glutamate toxicity (31). In addition, Müller glia is not only a source of neurotrophins (41) within the retina, but also respond to neurotrophins as they express all the neurotrophin receptors (41). Release of neurotrophins from Müller glia can also be promoted by other neurotrophins (42), inflammatory cytokines (43), as well by activated microglia (30). However, this response appears to be selective, as microglia conditioned media increases the Müller glia expression of mRNA coding for BDNF, but does not modify the expression of NGF, NT-3, CNTF (30). That Müller glia produces neurotrophins in response to selective neurotrophins is demonstrated by observations that binding of GDNF to the GFR $\alpha 1$ and GFR $\alpha 2$ increases their expression of BDNF, GDNF, and bFGF (30), and that binding of BDNF and GDNF to their corresponding receptors in Müller glia trigger a signalling cascade of activation that induces the production of neuronal survival factors, such as bFGF (44), demonstrating an additional mechanism by which Müller glia may promote neuroprotection.

NGF is a prototypic neurotrophin, with properties and functions that define the neurotrophic family of growth factor polypeptides. It signals with high-affinity through the TrkA receptor, and plays a pivotal role during the development, maintenance, and regeneration of sensory neurons in the CNS (45). It is highly expressed in the human eye during development and its mature form selectively binds to TrkA, promoting neural cell survival within the retina, in particular of retinal ganglion cells (RGCs) (46). In addition to its neurotrophic functions on retinal neurons, NGF has been shown to be a potent antioxidant, as demonstrated by its effect in preventing oxidant-induced lipid peroxide production by Müller glia in response to $\mathrm{H}_{2} \mathrm{O}_{2}$-induced oxidative stress (47).

\section{Multiple functions of neurotrophins within the retina}

BDNF shares similarities to NGF both in structure and function and is an important factor needed for the development and differentiation of retinal neurons (48). The importance of BDNF signalling continues into adulthood, where it is thought to be a major neurotrophin responsible for neuronal plasticity (49). Importantly, BDNF promotes the survival of neurons in the presence of harmful stimuli such as oxidative stress, and binding of exogenous BDNF to the truncated TrkB and p75 ${ }^{\text {NTR }}$ receptors in Müller glia induces upregulation of the expression of CNTF and bFGF (30). Culture of Müller cells in the presence of BDNF induces an increased expression of NESTIN, a neural stem/progenitor cell marker (50). During transient retinal ischemia, BDNF prevents osmotic swelling of Müller and bipolar cells by inducing activation of bFGF signalling (44). Furthermore, BDNF-TrkB signalling has been found to promote the regenerative potential of Müller glia by inducing Müller glial cell transdifferentiation into photoreceptor cells (50).

Less is known regarding the precise functions of NT-3 and NT-4 in the retina, in part due to their more recent discovery. NT4 preferentially binds TrkB, which is widely expressed by ganglion, horizontal, dopaminergic amacrine and Müller cells. In contrast, NT3 can bind to TrkA, TrkB and TrkC (51), but mainly binds to TrkC, which is preferentially expressed by photoreceptors and Müller cells (52). Treatment of Müller cells with exogenous NT-3 in culture increases their production of bFGF (41), whilst its neuroprotective effects on photoreceptors appears to be similar to that of BDNF (53). Recent studies in an inducible mouse model of Müller cell ablation showed that loss of Müller cells was associated with reduced expression of mature NT3 and upregulation of pro-NT3 and P75 NTR, further supporting the role of Müller glia in retinal neuroprotection (54). 
Although the neurotrophin family of molecules is the most well studied, there is compelling evidence that other factors have an important neuroprotective function within the retina and the CNS, and have been shown to be produced by Müller glia. These include ciliary neurotrophic factor (CNTF), glial cell-derived neurotrophic factor (GDNF) and pigment epithelium-derived factor (PEDF).

Ciliary neurotrophic factor (CNTF) is a molecule that is closely related to interleukin-6. It was initially characterized by its capacity to support the survival of chick embryo ciliary ganglion neurons in vitro, and was first isolated from the chick embryo choroid-iris-pigment epithelium complex and the adult rat sciatic nerve (55). CNTF is one of the most studied neurotrophins and has been shown to promote rod photoreceptor and cone outer segment regeneration in the degenerating retina, as well as axonal protection in RGCs (56). Extracellular CNTF binds to a receptor complex formed by CNTFR $\alpha$, gp130 and leukaemia inhibitory factor receptor beta (LIFRß) in Müller cells (57). This leads to activation of the JAK/Tyk kinase pathway and subsequently to STAT3 activation, a major downstream effector that controls gene transcription (57). Notably, Müller glia have been shown to upregulate their secretion of CNTF and FGF2 in response to light-induced retinal damage (58), and the effect of CNTF on RGC protection has been thought to be indirectly exerted by increased expression of GLAST1, glutamine synthetase, and connexin 43 by Müller glia (59). In addition to promoting photoreceptor cell survival, CNTF also reversibly reduces rhodopsin expression, causing shortening of rod outer segments and suppression of electroretinogram responses (60). The mechanisms behind these findings are not fully understood, but there is some evidence to suggest that the effect of CNTF on photoreceptors may be exerted via activation of Müller glia, which are the major cells in the retina that exhibit STAT3 phosphorylation in response to CNTF (57).

The glial cell line-derived neurotrophic factor (GDNF) family of ligands include GDNF, neurturin (NTN), artemin (ARTN), and persephin (PSPN). Although not an archetype neurotrophin, GDNF signal in a manner similar to neurotrophins (61). They bind to the family of receptors ligands GFR $\alpha 1$ and GFR $\alpha 2$ and their complexes signal through the transmembrane RET receptor tyrosine kinase (62). In accordance with the neurotrophin family functions, GDNF has been shown to protect photoreceptors from damage in animal models of retinal degeneration (63). Müller glia not only release GDNF, but also express GFR $\alpha 1$ and GFR 2 receptors. Exposure of these cells to high levels of glucose induce their release of GDNF, which protect them from apoptosis. On this basis it has been suggested that GDNF may have a protective role in Müller cells survival during the early stages of diabetic retinopathy (64).

PEDF is a member of the serine protease inhibitor family. Within the retina, this factor is produced by RPE cells and Müller glia (65). The receptor for PEDF is a phospholipase membrane protein, which is expressed by retinal neuronal cells, with the highest concentration found within photoreceptor inner segments (65). The neuroprotective effect of PEDF has been demonstrated by observations that injection of PEDF protein peptides into the eye of the $\mathrm{C} 3 \mathrm{H} / \mathrm{HeN}$ retinal degeneration $1(r d 1)$ mouse confers strong neuroprotection to degenerating photoreceptors (66). This rescue from photoreceptor cell death has been attributed to a decrease in intracellular $\mathrm{Ca}^{2+}$ concentrations and consequently to a decrease in calpain activity induced by PEDF (67). Further confirmation of the neurotrophic effects of PEDF have been shown by inhibition of photoreceptor apoptosis following lentivirus-mediated retinal gene transfer of PEDF into the subretinal space of the RCS rat. This effect has been associated with a decrease in nuclear translocation of the apoptosis-inducing factor (AIF) and an increase of the B-cell lymphoma 2 (BCL2) protein within the retina (68). 


\section{Müller glia as a source of antioxidants in the neural retina}

Due to the high metabolic functions of the retina that are required for the visual process, there is a great need for antioxidant protection in this tissue (69). Much of the literature related to oxidative stress in the retina, has understated the important antioxidant role of Müller glia, and has focused on their production of oxidative products, such as nitric oxide synthase (NOS II), which have a detrimental effect on retinal neurons (70). Oxidative stress is often associated with major retinal degenerative diseases such as glaucoma, age related macular degeneration (AMD), diabetic retinopathy (DR) and retinitis pigmentosa. Müller cells undoubtedly contribute to oxidative stress due to malfunctions of glutamate uptake and glutathione synthesis in these conditions (71). They also play a crucial role in preventing or neutralising oxidative stress due to their inherent metabolic functions that provide potent antioxidant factors to prevent neural damage. For example, glutamate transporters, which are highly expressed by Müller glia, prevent excitotoxic retinal damage by promoting the synthesis of glutathione, a tripeptide synthesized from glutamate, cysteine and glycine (72). This has been recognized as a major retinal antioxidant released by Müller glia in response to hypoxia and oxidative stress, and plays a critical role in the control of harmful oxidative species in the neural retina (71). Glutathione has been shown to prevent RGC degeneration in experimental models of glaucoma (73).

\section{Antioxidant gene transcription regulated by the nuclear factor erythroid 2-related factor 2 (NRF2)}

In response to oxidative stress, Müller cells have been shown to upregulate mRNA expression of the transcription factor nuclear factor erythroid 2-related factor 2 (NRF2), an important antioxidant molecule that regulates transcription of more than 100 antioxidant/cytoprotective genes $(74,75)$. NRF2 is also a potent inductor of glutathione release, which as indicated above, is a major antioxidant produced by Müller glia in vivo and in vitro. In cultured Müller glia, NRF2 inhibition significantly decreases antioxidant gene expression and exacerbates induced oxidative stress, whilst its activation suppresses oxidant-induced reactive oxygen species (76).

The NRF2 gene binds to DNA response elements, known as known as antioxidant response elements (ARE) and regulates the expression of genes involved in the response to cellular stress, such as phase 2 enzymes, which include the nicotinamide adenine dinucleotide phosphatase (NADPH)-quinone oxidoreductase (NQO-1), heme oxygenase-1 (HO-1), superoxide dismutase2 (SOD2), catalase (CAT) and glutathione peroxidase 1 (GTX1) (75). NRF2 has been shown to reduce levels of reactive oxygen species by directly controlling the enzymatic formation of nitric oxide synthase NOS2, regulating levels of $\mathrm{H}_{2} \mathrm{O}_{2}$ and promoting synthesis of SOD and CAT, the enzymes responsible for the breakdown of $\mathrm{H}_{2} \mathrm{O}_{2}$ into hydrogen and oxygen (59). Association between antioxidant suppression and diabetic induced retinal dysfunction has been shown in the Nrf2 knockout diabetic mice. In this model, mice exhibit reduced retinal glutathione and increased TNF- $\alpha$ protein levels, compared with wild-type mice. This is associated with an early onset of blood-retina barrier damage, significant increase in superoxide levels and exacerbation of neuronal dysfunction (76).

Recent proteomic studies of isolated Müller cells have identified their expression of several enzymes involved in oxidative defence mechanisms, including the glutathione $S$ transferases Mu1 and Mu5 (GSTM1 and 5), paraoxonase/arylesterase 2 (PON2), and peroxiredoxins 1, 4 and 6 (PRDX1, 4 and 6 ) (77). These observations have significantly contributed to the understanding of the antioxidant 
functions of Müller glia that confer retinal neuroprotection in response to oxidative mechanisms triggered by disease or injury. This is further supported by findings that Müller glia in culture increase their expression of the antioxidant genes NQO-1, CAT, SOD2, HO-1 and GPX1 in response to homocysteine (75), an excitatory amino acid implicated in RGCs loss that is associated with glaucoma (78). The involvement of GSTs has been demonstrated in antioxidant neuroprotective mechanisms observed in AMD and DR. For instance, mRNA levels of GSTM1 and GSTM5 are significantly decreased in the neurosensory retina of post-mortem AMD eyes when compared with normal tissue (79), and this decrease has been associated to hyper-methylation of the GSTM1 promoter, suggesting that GSTM1 and GSTM5 undergo epigenetic repression in AMD, which may increase susceptibility to oxidative stress in this condition (79). Furthermore, meta-analysis performed to assess the association between GSTM1/GSTT1 deletions and DR risk, has shown that increased risk of DR is associated with the null polymorphism of these two genes and consequently higher oxidative stress (80).

Peroxiredoxin 6 (PRDX6) is a key regulator of the cellular redox balance that has the ability to neutralize peroxides, peroxynitrite, and phospholipid hydroperoxides with the use of electrons from thioredoxin (81). Among the six members of this peroxidase family, PRDX6 is highly expressed in mammalian Müller cells (77) and is the only member that has both, peroxide and phospholipase $A_{2}$ activity and its activity is dependent upon binding of phospholipids (82). Supplementation of PRDX6 has been shown to attenuate reactive oxygen species and TGFB-induced insult to glaucomatous trabecular meshwork cells in vitro, as well as to decrease the senescence process in these cells (83). Moreover, the glaucomatous neuroprotective effects of this antioxidant factor have been demonstrated on RGC, as demonstrated by observations that reactive oxygen species-mediated suppression of PRDX6 in these cells can be prevented by over-expression of PRDX6 (84). Observations that high levels of PRDX6 are expressed by Müller glia further support the antioxidant role of these cells within the retina. Production of this molecule by Müller glia upon retinal injury may limit the damage caused by reactive oxygen species on retinal neurons and may play a major neuroprotective role in many retinal degenerative diseases such as glaucoma and macular degeneration. However, further studies are much needed to elucidate the neuroprotective role of PRDX6 during retinal disease.

Superoxide dismutases (SODs) are enzymes that protect against oxidative stress by dismutation of superoxide into oxygen and hydrogen peroxide through cyclic reduction and oxidation of iron or manganese. SOD2, also known as MnSOD is found in the mitochondrial matrix and protects against the superoxide generated by electron leakage from the electron-transport chain (85). The activity of SOD2 requires magnesium and it actively binds to superoxide byproducts of oxidative phosphorylation such as hydrogen radical $\left(\mathrm{H}^{+}\right)$, converting them into hydrogen peroxide and diatomic oxygen (85). Human SOD2 is translated as a precursor peptide with a leader sequence that is proteolytically removed by matrix metalloproteinases in the mitochondrial matrix during the processing of the mature enzyme (85). It is widely expressed in most tissues and abundant in trabecular meshwork cells (86). Dietary supplementation of SOD to a rat model of glaucoma has shown that SOD exerts an anti-apoptotic effect on RGCs against oxidative stress induced by ischemia and ocular hypertension (87), and RPE-specific deletion of SOD2 in a mouse model of AMD results in increased oxidative stress and disruption of RPE functions (88). 
HO-1, also known as heat shock protein 32 (HSP32) forms another defense mechanism against oxidative stress. As indicated by its alternative name, $\mathrm{HO}-1$ has a heat shock regulatory element. It also has additional regulatory elements in the promoter region. These elements bind to their respective inducers and cause transcription of the gene (89). HO-1 is inducible by a wide range of stimuli known to cause oxidative stress, such as hyperoxia, hypoxia, heat shock, endotoxins, cytokines and nitric oxide. HO-1 is involved in the heme catabolism and acts by cleaving the heme ring at the alpha methene bridge to produce the biologically active end products biliverdin, $\mathrm{CO}$ and ferrous ion (89). Various studies have demonstrated that the HO-1/CO system exhibits potent anti-oxidative, antiapoptotic, anti-inflammatory and cytoprotective activities against ischaemia-reperfusion injury, indicating a potential therapeutic use of these molecules to treat diseases associated with this type of injury (89). The transcription of HO-1 is regulated by NRF2 as evidenced in the Nrf2 knockout mice, which shows elevated oxidative stress that leads to RPE degeneration (90). Müller glia has been shown to express HO-1 protein, and its production is significantly upregulated upon exposure to advanced lipoxidation end products (91), suggesting that these products trigger a neuroprotective response by Müller cells within the retina.

\section{Potential of Müller glia as a vehicle to deliver neuroprotective factors into the retina}

Despite a large body of data which have demonstrated the neuroprotective features of neurotrophins and antioxidants, delivery of these molecules into the eye remains a major challenge in translating our current knowledge into clinical practice. Direct injections of neurotrophins into the vitreous are not practical given their low bioavailability and short half-life, for which gene therapies, using adenoviral, adeno-associated (AVV) and lentiviral vectors as methods to deliver these molecules into the retina have been actively explored (92); however, gene therapies so far have been limited to the introduction of single molecules, and effective neuroprotection of the retina may not be achieved by either injection of individual trophic factors or gene therapies targeting single neurotrophins. An example of these approaches is illustrated by early studies that utilized intraocular implants of encapsulated cells genetically modified to secrete CNTF. Whilst studies in the rcd1 canine model of retinitis pigmentosa showed photoreceptor rescue by these implants (93), and early clinical trials showed safety and tolerability (94), this approach appeared not to have a long lasting therapeutic benefit and there is no documentation of this therapy having been implemented (95). Other methods have employed eye drops to deliver NGF into the eye (96), but no advances have been published nor does the method appear to have been adopted in retinal therapies since its early clinical trials in 2014. More recent experimental approaches have utilized intravitreal AAV gene delivery to increased expression of both BDNF and its TrkB receptor by RGC and amacrine cells (97). This innovative approach may constitute an effective method for inducing neuroprotection in glaucoma, but further studies are yet to confirm the therapeutic value in patients with glaucoma.

Intravitreal injection of Müller glia with stem cell characteristics into experimental models of RGC and photoreceptor cell damage has resulted in partial recovery of retinal function, and in the absence of retinal cell integration, this effect can only be attributed to the neuroprotective functions of these cells(98-100). Cellular approaches utilizing Müller glia may have the potential to by-pass many of the current issues of neurotrophin delivery, with cells offering the advantage of being able to deliver a wide range of neuroprotective factors into the retinal microenvironment (summarized in Fig. 2). They are naturally homeostatic and responsive to change, and their continuous production of these factors may potentially prolong the bio-availability of neuroprotectant molecules within the damaged retina. Although this approach presents its own challenges due to the regulatory requirements for cell isolation, expansion and histocompatibility matching, Müller glia constitute an attractive source for 
use in cell therapies as neuroprotective factors released by these cells may act in concert with each other to provide lasting protection of retinal neurons.

\section{Conclusions and future trends}

Retinal regeneration is a major area of intensive research as there is an urgent need to prevent blindness and restore sight lost by degenerative diseases. Recent studies in the field have been focused on the implementation of stem cell transplantation to replace damaged neurons, but despite many efforts, neural replacement by cell grafting has not been achieved and still requires much exploration. Based on evidence that Müller glia with stem cell characteristics are present in the adult human retina, induction of neural cell renewal by resident Müller cells may potentially constitute a practical approach to regeneration in preference to transplantation. However, this concept is only just emerging and will require extensive studies before self-repair of the neural retina can be achieved. Other approaches to induce retina regeneration using neurotrophin injections into the eye have not proved successful or long lasting in clinical trials, and new technologies involving gene therapies to induce overexpression of neurotrophins and their regulatory molecules still need clinical development. Due to the inherent metabolic functions of Müller glia, which are neuroprotective in nature, intraocular transplantation of these cells as a source of neurotrophins and neuroprotective antioxidant molecules may constitute an alternative approach to promote retina repair and regeneration, and the therapeutic potential of these cells merits further consideration.

\section{Acknowledgements}

The authors were supported by the MRC grant Ref MR/P01660X/1; Moorfields Eye Charity, Fight for Sight and the NIHR Biomedical Research Centre at Moorfields Eye Hospital and UCL Institute of Ophthalmology, London, UK. 


\section{Figure legends}

Fig. 1. Anatomical distribution of Müller glia within the retina: Representative illustration shows the anatomical distribution of Müller glia within the neural retina. Müller glia (red) span the entire retina and make contact with all retinal neurons. Their soma lay in the inner nuclear layer and processes expand to the outer retinal layers and inner retinal layers. ONL=outer nuclear layer; $O P L=0 u t e r$ plexiform layer; $\mathrm{INL}=$ inner nuclear layer; $\mathrm{IPL}=$ inner plexiform layer; $\mathrm{GCL}=$ ganglion cell layer.

Fig.2. Multiple neuroprotective functions of Müller glia: The inherent metabolic functions of Müller glia are themselves neuroprotective in nature. Neuroprotection by these cells is further potentiated by their release of neurotrophins and antioxidants into the retinal microenvironment. 


\section{References}

1. Charlton-Perkins M, Almeida AD, MacDonald RB, Harris WA. Genetic control of cellular morphogenesis in Muller glia. Glia. 2019;67(7):1401-11.

2. Huang EJ, Reichardt LF. Neurotrophins: roles in neuronal development and function. Annu Rev Neurosci. 2001;24:677-736.

3. MacDonald RB, Randlett O, Oswald J, Yoshimatsu T, Franze K, Harris WA. Muller glia provide essential tensile strength to the developing retina. J Cell Biol. 2015;210(7):1075-83.

4. Byrne LC, Khalid F, Lee T, Zin EA, Greenberg KP, Visel M, Schaffer DV, Flannery JG. AAVmediated, optogenetic ablation of Muller Glia leads to structural and functional changes in the mouse retina. PLoS One. 2013;8(9):e76075.

5. Chung SH, Gillies M, Sugiyama Y, Zhu L, Lee SR, Shen W. Profiling of microRNAs involved in retinal degeneration caused by selective Muller cell ablation. PLoS One. 2015;10(3):e0118949.

6. Wang J, O'Sullivan ML, Mukherjee D, Punal VM, Farsiu S, Kay JN. Anatomy and spatial organization of Muller glia in mouse retina. J Comp Neurol. 2017;525(8):1759-77.

7. Chao TI, Grosche J, Friedrich KJ, Biedermann B, Francke M, Pannicke T, Reichelt W, Wulst M, Mühle C, Pritz-Hohmeier S, et al. Comparative studies on mammalian Muller (retinal glial) cells. J Neurocytol. 1997;26(7):439-54.

8. Robinson SR, Dreher Z. Muller cells in adult rabbit retinae: morphology, distribution and implications for function and development. J Comp Neurol. 1990;292(2):178-92.

9. Uga S, Smelser. Comparative study of the fine structure of retinal Muller cells in various vertebrates. Invest Ophthalmol. 1973;12(6):434-48.

10. Slezak M, Grosche A, Niemiec A, Tanimoto N, Pannicke T, Munch TA, Münch TA, Crocker B, Isope $P$, Härtig $W$, et al. Relevance of exocytotic glutamate release from retinal glia. Neuron. 2012;74(3):504-16.

11. Williams DS, Arikawa K, Paallysaho T. Cytoskeletal components of the adherens junctions between the photoreceptors and the supportive Muller cells. J Comp Neurol. 1990;295(1):155-64.

12. Bringmann A, Pannicke T, Grosche J, Francke M, Wiedemann P, Skatchkov SN, Osborne NN, Reichenbach A. Muller cells in the healthy and diseased retina. Prog Retin Eye Res. 2006;25(4):397424.

13. Derouiche A, Rauen T. Coincidence of L-glutamate/L-aspartate transporter (GLAST) and glutamine synthetase (GS) immunoreactions in retinal glia: evidence for coupling of GLAST and GS in transmitter clearance. J Neurosci Res. 1995;42(1):131-43.

14. Izumi Y, Kirby CO, Benz AM, Olney JW, Zorumski CF. Muller cell swelling, glutamate uptake, and excitotoxic neurodegeneration in the isolated rat retina. Glia. 1999;25(4):379-89.

15. Pow DV, Robinson SR. Glutamate in some retinal neurons is derived solely from glia. Neuroscience. 1994;60(2):355-66.

16. Rauen T, Rothstein JD, Wassle H. Differential expression of three glutamate transporter subtypes in the rat retina. Cell Tissue Res. 1996;286(3):325-36.

17. Pow DV, Barnett NL. Changing patterns of spatial buffering of glutamate in developing rat retinae are mediated by the Muller cell glutamate transporter GLAST. Cell Tissue Res. 1999;297(1):5766.

18. Sarthy VP, Pignataro L, Pannicke T, Weick M, Reichenbach A, Harada T, Tanaka K, Marc R. Glutamate transport by retinal Muller cells in glutamate/aspartate transporter-knockout mice. Glia. 2005;49(2):184-96.

19. Harada T, Harada C, Watanabe M, Inoue Y, Sakagawa T, Nakayama N, Sasaki S, Okuyama S, Watase K, Wada K, et al. Functions of the two glutamate transporters GLAST and GLT-1 in the retina. Proc Natl Acad Sci U S A. 1998;95(8):4663-6.

20. Marshak DW. A tale of two neurotransmitters. Vis Neurosci. 2016;33:E017.

21. Biedermann B, Bringmann A, Franze K, Faude F, Wiedemann P, Reichenbach A. GABA(A) receptors in Muller glial cells of the human retina. Glia. 2004;46(3):302-10. 
22. Biedermann B, Bringmann A, Reichenbach A. High-affinity GABA uptake in retinal glial (Muller) cells of the guinea pig: electrophysiological characterization, immunohistochemical localization, and modeling of efficiency. Glia. 2002;39(3):217-28.

23. Pfeiffer-Guglielmi B, Francke M, Reichenbach A, Fleckenstein B, Jung G, Hamprecht B. Glycogen phosphorylase isozyme pattern in mammalian retinal Muller (glial) cells and in astrocytes of retina and optic nerve. Glia. 2005;49(1):84-95.

24. Hurley JB, Lindsay KJ, Du J. Glucose, lactate, and shuttling of metabolites in vertebrate retinas. J Neurosci Res. 2015;93(7):1079-92.

25. Poitry-Yamate CL, Poitry S, Tsacopoulos M. Lactate released by Muller glial cells is metabolized by photoreceptors from mammalian retina. J Neurosci. 1995;15(7 Pt 2):5179-91.

26. Tada $\mathrm{Y}$, Horio $\mathrm{Y}$, Kurachi $\mathrm{Y}$. Inwardly rectifying $\mathrm{K}+$ channel in retinal Muller cells: comparison with the KAB-2/Kir4.1 channel expressed in HEK293T cells. Jpn J Physiol. 1998;48(1):71-80.

27. Pannicke T, Ivo Chao T, Reisenhofer M, Francke $M$, Reichenbach A. Comparative electrophysiology of retinal Muller glial cells-A survey on vertebrate species. Glia. 2017;65(4):533-68.

28. Bringmann A, Wiedemann P. Muller glial cells in retinal disease. Ophthalmologica. 2012;227(1):1-19.

29. Eastlake K, Banerjee PJ, Angbohang A, Charteris DG, Khaw PT, Limb GA. Muller glia as an important source of cytokines and inflammatory factors present in the gliotic retina during proliferative vitreoretinopathy. Glia. 2016;64(4):495-506.

30. Harada T, Harada C, Kohsaka S, Wada E, Yoshida K, Ohno S, Mamada H, Tanaka K, Parada LF, Wada K. Microglia-Muller glia cell interactions control neurotrophic factor production during lightinduced retinal degeneration. J Neurosci. 2002;22(21):9228-36.

31. Taylor S, Srinivasan B, Wordinger RJ, Roque RS. Glutamate stimulates neurotrophin expression in cultured Muller cells. Brain Res Mol Brain Res. 2003;111(1-2):189-97.

32. Arai-Gaun S, Katai N, Kikuchi T, Kurokawa T, Ohta K, Yoshimura N. Heme oxygenase-1 induced in muller cells plays a protective role in retinal ischemia-reperfusion injury in rats. Invest Ophthalmol Vis Sci. 2004;45(11):4226-32.

33. Deliyanti D, Lee JY, Petratos S, Meyer CJ, Ward KW, Wilkinson-Berka JL, de Haan JB. A potent Nrf2 activator, dh404, bolsters antioxidant capacity in glial cells and attenuates ischaemic retinopathy. Clin Sci (Lond). 2016;130(15):1375-87.

34. Ceni C, Unsain N, Zeinieh MP, Barker PA. Neurotrophins in the regulation of cellular survival and death. Handb Exp Pharmacol. 2014;220:193-221.

35. West AE, Pruunsild P, Timmusk T. Neurotrophins: transcription and translation. Handb Exp Pharmacol. 2014;220:67-100.

36. Meldolesi J. Neurotrophin receptors in the pathogenesis, diagnosis and therapy of neurodegenerative diseases. Pharmacol Res. 2017;121:129-37.

37. Skaper SD. The biology of neurotrophins, signalling pathways, and functional peptide mimetics of neurotrophins and their receptors. CNS Neurol Disord Drug Targets. 2008;7(1):46-62.

38. Chao MV. Neurotrophins and their receptors: a convergence point for many signalling pathways. Nat Rev Neurosci. 2003;4(4):299-309.

39. Sun XL, Chen BY, Duan L, Xia Y, Luo ZJ, Wang JJ, Rao ZR, Chen LW. The proform of glia cell linederived neurotrophic factor: a potentially biologically active protein. Mol Neurobiol. 2014;49(1):23450.

40. Bruno MA, Cuello AC. Activity-dependent release of precursor nerve growth factor, conversion to mature nerve growth factor, and its degradation by a protease cascade. Proc Natl Acad Sci U S A. 2006;103(17):6735-40.

41. Harada T, Harada C, Nakayama N, Okuyama S, Yoshida K, Kohsaka S, Matsuda H, Wada K. Modification of glial-neuronal cell interactions prevents photoreceptor apoptosis during light-induced retinal degeneration. Neuron. 2000;26(2):533-41.

42. Wahlin KJ, Campochiaro PA, Zack DJ, Adler R. Neurotrophic factors cause activation of intracellular signaling pathways in Muller cells and other cells of the inner retina, but not photoreceptors. Invest Ophthalmol Vis Sci. 2000;41(3):927-36. 
43. Boss JD, Singh PK, Pandya HK, Tosi J, Kim C, Tewari A, Juzych MS, Abrams GW, Kumar A. Assessment of Neurotrophins and Inflammatory Mediators in Vitreous of Patients With Diabetic Retinopathy. Invest Ophthalmol Vis Sci. 2017;58(12):5594-603.

44. Berk BA, Vogler S, Pannicke T, Kuhrt H, Garcia TB, Wiedemann P, Reichenbach A, Seeger J, Bringmann A. Brain-derived neurotrophic factor inhibits osmotic swelling of rat retinal glial (Muller) and bipolar cells by activation of basic fibroblast growth factor signaling. Neuroscience. 2015;295:175-86.

45. Roberti G, Mantelli F, Macchi I, Massaro-Giordano M, Centofanti M. Nerve growth factor modulation of retinal ganglion cell physiology. J Cell Physiol. 2014;229(9):1130-3.

46. Lee R, Kermani P, Teng KK, Hempstead BL. Regulation of cell survival by secreted proneurotrophins. Science. 2001;294(5548):1945-8.

47. Giardino I, Fard AK, Hatchell DL, Brownlee M. Aminoguanidine inhibits reactive oxygen species formation, lipid peroxidation, and oxidant-induced apoptosis. Diabetes. 1998;47(7):1114-20. 48. Jacobson MD, Weil M, Raff MC. Programmed cell death in animal development. Cell. 1997;88(3):347-54.

49. Mandolesi G, Menna E, Harauzov A, von Bartheld CS, Caleo M, Maffei L. A role for retinal brain-derived neurotrophic factor in ocular dominance plasticity. Curr Biol. 2005;15(23):2119-24. 50. Harada C, Guo X, Namekata K, Kimura A, Nakamura K, Tanaka K, Parada LF, Harada T. Gliaand neuron-specific functions of TrkB signalling during retinal degeneration and regeneration. Nat Commun. 2011;2:189.

51. Bothwell M. Functional interactions of neurotrophins and neurotrophin receptors. Annu Rev Neurosci. 1995;18:223-53.

52. Friedman WJ. Proneurotrophins, seizures, and neuronal apoptosis. Neuroscientist. 2010;16(3):244-52.

53. LaVail MM, Unoki K, Yasumura D, Matthes MT, Yancopoulos GD, Steinberg RH. Multiple growth factors, cytokines, and neurotrophins rescue photoreceptors from the damaging effects of constant light. Proc Natl Acad Sci U S A. 1992;89(23):11249-53.

54. Shen W, Zhu L, Lee SR, Chung SH, Gillies MC. Involvement of NT3 and P75(NTR) in photoreceptor degeneration following selective Muller cell ablation. J Neuroinflammation. 2013;10:137.

55. Adler R. Ciliary neurotrophic factor as an injury factor. Curr Opin Neurobiol. 1993;3(5):785-9.

56. Wen R, Tao W, Li Y, Sieving PA. CNTF and retina. Prog Retin Eye Res. 2012;31(2):136-51.

57. Rhee KD, Nusinowitz S, Chao K, Yu F, Bok D, Yang XJ. CNTF-mediated protection of photoreceptors requires initial activation of the cytokine receptor gp130 in Muller glial cells. Proc Natl Acad Sci U S A. 2013;110(47):E4520-9.

58. Joly S, Pernet V, Chemtob S, Di Polo A, Lachapelle P. Neuroprotection in the juvenile rat model of light-induced retinopathy: evidence suggesting a role for FGF-2 and CNTF. Invest Ophthalmol Vis Sci. 2007;48(5):2311-20.

59. van Adel BA, Arnold JM, Phipps J, Doering LC, Ball AK. Ciliary neurotrophic factor protects retinal ganglion cells from axotomy-induced apoptosis via modulation of retinal glia in vivo. J Neurobiol. 2005;63(3):215-34.

60. Wen R, Song Y, Liu Y, Li Y, Zhao L, Laties AM. CNTF negatively regulates the phototransduction machinery in rod photoreceptors: implication for light-induced photostasis plasticity. Adv Exp Med Biol. 2008;613:407-13.

61. Poo MM. Neurotrophins as synaptic modulators. Nat Rev Neurosci. 2001;2(1):24-32.

62. Airaksinen MS, Saarma M. The GDNF family: signalling, biological functions and therapeutic value. Nat Rev Neurosci. 2002;3(5):383-94.

63. Garcia-Caballero C, Lieppman B, Arranz-Romera A, Molina-Martinez IT, Bravo-Osuna I, Young $M$, Baranov $P$, Herrero-Vanrell R. Photoreceptor preservation induced by intravitreal controlled delivery of GDNF and GDNF/melatonin in rhodopsin knockout mice. Mol Vis. 2018;24:733-45. 
64. Zhu X, Sun Y, Wang Z, Cui W, Peng Y, Li R. Expression of glial cell line-derived neurotrophic factor and its receptors in cultured retinal Muller cells under high glucose circumstance. Anat Rec (Hoboken). 2012;295(3):532-9.

65. Yafai Y, Lange J, Wiedemann P, Reichenbach A, Eichler W. Pigment epithelium-derived factor acts as an opponent of growth-stimulatory factors in retinal glial-endothelial cell interactions. Glia. 2007;55(6):642-51.

66. Kenealey J, Subramanian P, Comitato A, Bullock J, Keehan L, Polato F, Hoover D, Marigo V, Becerra SP. Small Retinoprotective Peptides Reveal a Receptor-binding Region on Pigment Epithelium-derived Factor. J Biol Chem. 2015;290(42):25241-53.

67. Comitato A, Subramanian P, Turchiano G, Montanari M, Becerra SP, Marigo V. Pigment epithelium-derived factor hinders photoreceptor cell death by reducing intracellular calcium in the degenerating retina. Cell Death Dis. 2018;9(5):560.

68. Murakami Y, Ikeda Y, Yonemitsu Y, Onimaru M, Nakagawa K, Kohno R, Miyazaki M, Hisatomi $T$, Nakamura M, Yabe T, et al. Inhibition of nuclear translocation of apoptosis-inducing factor is an essential mechanism of the neuroprotective activity of pigment epithelium-derived factor in a rat model of retinal degeneration. Am J Pathol. 2008;173(5):1326-38.

69. Yu DY, Cringle SJ. Retinal degeneration and local oxygen metabolism. Exp Eye Res. 2005;80(6):745-51.

70. Cotinet A, Goureau O, Hicks D, Thillaye-Goldenberg B, de Kozak Y. Tumor necrosis factor and nitric oxide production by retinal Muller glial cells from rats exhibiting inherited retinal dystrophy. Glia. 1997;20(1):59-69.

71. Pow DV, Crook DK. Immunocytochemical evidence for the presence of high levels of reduced glutathione in radial glial cells and horizontal cells in the rabbit retina. Neurosci Lett. 1995;193(1):258.

72. Reichelt W, Stabel-Burow J, Pannicke T, Weichert $\mathrm{H}$, Heinemann U. The glutathione level of retinal Muller glial cells is dependent on the high-affinity sodium-dependent uptake of glutamate. Neuroscience. 1997;77(4):1213-24.

73. Harada T, Harada C, Nakamura K, Quah HM, Okumura A, Namekata K, Saeki T, Aihara M, Yoshida $\mathrm{H}$, Mitani A, et al. The potential role of glutamate transporters in the pathogenesis of normal tension glaucoma. J Clin Invest. 2007;117(7):1763-70.

74. Inoue $Y$, Shimazawa M, Noda Y, Nagano R, Otsuka T, Kuse Y, Nakano Y, Tsuruma K, Nakagami $\mathrm{Y}, \mathrm{Hara}$ H. RS9, a novel Nrf2 activator, attenuates light-induced death of cells of photoreceptor cells and Muller glia cells. J Neurochem. 2017;141(5):750-65.

75. Navneet S, Cui X, Zhao J, Wang J, Kaidery NA, Thomas B, Bollinger KE, Yoon Y, Smith SB. Excess homocysteine upregulates the NRF2-antioxidant pathway in retinal Muller glial cells. Exp Eye Res. 2019;178:228-37.

76. Xu Z, Wei Y, Gong J, Cho H, Park JK, Sung ER, et al. NRF2 plays a protective role in diabetic retinopathy in mice. Diabetologia. 2014;57(1):204-13.

77. Grosche A, Hauser A, Lepper MF, Mayo R, von Toerne C, Merl-Pham J, Hauck SM. The Proteome of Native Adult Muller Glial Cells From Murine Retina. Mol Cell Proteomics. 2016;15(2):462-80.

78. Roedl JB, Bleich S, Reulbach U, von Ahsen N, Schlotzer-Schrehardt U, Rejdak R, Naumann GO, Kruse FE, Kornhuber J, Jünemann AG. Homocysteine levels in aqueous humor and plasma of patients with primary open-angle glaucoma. J Neural Transm (Vienna). 2007;114(4):445-50.

79. Hunter A, Spechler PA, Cwanger A, Song Y, Zhang Z, Ying GS, Hunter AK, Dezoeten E, Dunaief JL. DNA methylation is associated with altered gene expression in AMD. Invest Ophthalmol Vis Sci. 2012;53(4):2089-105.

80. Sun L, Zhang Y, Xiong Y. GSTM1 and GSTT1 null genotype and diabetic retinopathy: a metaanalysis. Int J Clin Exp Med. 2015;8(2):1677-83.

81. Pacifici F, Arriga R, Sorice GP, Capuani B, Scioli MG, Pastore D, Donadel G, Bellia A, Caratelli S, Coppola A, Ferrelli F, Federici $M$, et al. Peroxiredoxin 6, a novel player in the pathogenesis of diabetes. Diabetes. 2014;63(10):3210-20. 
82. Fisher AB. Peroxiredoxin 6: a bifunctional enzyme with glutathione peroxidase and phospholipase A(2) activities. Antioxid Redox Signal. 2011;15(3):831-44.

83. Fatma N, Kubo E, Toris CB, Stamer WD, Camras CB, Singh DP. PRDX6 attenuates oxidative stress- and TGFbeta-induced abnormalities of human trabecular meshwork cells. Free Radic Res. 2009;43(9):783-95.

84. Tulsawani R, Kelly LS, Fatma N, Chhunchha B, Kubo E, Kumar A, Singh DP. Neuroprotective effect of peroxiredoxin 6 against hypoxia-induced retinal ganglion cell damage. BMC Neurosci. 2010;11:125.

85. Candas D, Li JJ. MnSOD in oxidative stress response-potential regulation via mitochondrial protein influx. Antioxid Redox Signal. 2014;20(10):1599-617.

86. Joe MK, Nakaya N, Abu-Asab M, Tomarev SI. Mutated myocilin and heterozygous Sod2 deficiency act synergistically in a mouse model of open-angle glaucoma. Hum Mol Genet. 2015;24(12):3322-34.

87. Nebbioso M, Scarsella G, Tafani M, Pescosolido N. Mechanisms of ocular neuroprotection by antioxidant molecules in animal models. J Biol Regul Homeost Agents. 2013;27(1):197-209.

88. Biswal MR, Ildefonso CJ, Mao H, Seo SJ, Wang Z, Li H, Le YZ, Lewin AS. Conditional Induction of Oxidative Stress in RPE: A Mouse Model of Progressive Retinal Degeneration. Adv Exp Med Biol. 2016;854:31-7.

89. Cheng $Y$, Rong J. Therapeutic Potential of Heme Oxygenase-1/carbon Monoxide System Against Ischemia-Reperfusion Injury. Curr Pharm Des. 2017;23(26):3884-98.

90. Pittala V, Fidilio A, Lazzara F, Platania CBM, Salerno L, Foresti R, Drago F, Bucolo C. Effects of Novel Nitric Oxide-Releasing Molecules against Oxidative Stress on Retinal Pigmented Epithelial Cells. Oxid Med Cell Longev. 2017;2017:1420892.

91. Yong PH, Zong H, Medina RJ, Limb GA, Uchida K, Stitt AW, Curtis TM. Evidence supporting a role for $\mathrm{N}$-(3-formyl-3,4-dehydropiperidino)lysine accumulation in Muller glia dysfunction and death in diabetic retinopathy. Mol Vis. 2010;16:2524-38.

92. Ghasemi M, Alizadeh E, Saei Arezoumand K, Fallahi Motlagh B, Zarghami N. Ciliary neurotrophic factor (CNTF) delivery to retina: an overview of current research advancements. Artif Cells Nanomed Biotechnol. 2018;46(8):1694-707.

93. Tao W, Wen R, Goddard MB, Sherman SD, O'Rourke PJ, Stabila PF, Bell WJ, Dean BJ, Kauper $K A, B u d z V A$, et al. Encapsulated cell-based delivery of CNTF reduces photoreceptor degeneration in animal models of retinitis pigmentosa. Invest Ophthalmol Vis Sci. 2002;43(10):3292-8.

94. Sieving PA, Caruso RC, Tao W, Coleman HR, Thompson DJ, Fullmer KR, Bush RA. Ciliary neurotrophic factor (CNTF) for human retinal degeneration: phase I trial of CNTF delivered by encapsulated cell intraocular implants. Proc Natl Acad Sci U S A. 2006;103(10):3896-901.

95. Birch DG, Weleber RG, Duncan JL, Jaffe GJ, Tao W. Ciliary Neurotrophic Factor Retinitis Pigmentosa Study G. Randomized trial of ciliary neurotrophic factor delivered by encapsulated cell intraocular implants for retinitis pigmentosa. Am J Ophthalmol. 2013;156(2):283-92 e1.

96. Ferrari MP, Mantelli F, Sacchetti M, Antonangeli MI, Cattani F, D'Anniballe G, Sinigaglia F, Ruffini PA, Lambiase A. Safety and pharmacokinetics of escalating doses of human recombinant nerve growth factor eye drops in a double-masked, randomized clinical trial. BioDrugs. 2014;28(3):275-83. 97. Osborne A, Khatib TZ, Songra L, Barber AC, Hall K, Kong GYX, Widdowson PS, Martin KR. Neuroprotection of retinal ganglion cells by a novel gene therapy construct that achieves sustained enhancement of brain-derived neurotrophic factor/tropomyosin-related kinase receptor-B signaling. Cell Death Dis. 2018;9(10):1007.

98. Becker S, Eastlake K, Jayaram H, Jones MF, Brown RA, McLellan GJ, McLellan GJ, Charteris DG, Khaw PT, Limb GA. Allogeneic Transplantation of Muller-Derived Retinal Ganglion Cells Improves Retinal Function in a Feline Model of Ganglion Cell Depletion. Stem Cells Transl Med. 2016;5(2):192205.

99. Eastlake K, Wang W, Jayaram H, Murray-Dunning C, Carr AJF, Ramsden CM, Vugler A, Gore K, Clemo N, Stewart M, et al. Phenotypic and Functional Characterization of Muller Glia Isolated from 
Induced Pluripotent Stem Cell-Derived Retinal Organoids: Improvement of Retinal Ganglion Cell Function upon Transplantation. Stem Cells Transl Med. 2019.

100. Jayaram H, Jones MF, Eastlake K, Cottrill PB, Becker S, Wiseman J, Khaw PT, Limb GA. Transplantation of photoreceptors derived from human Muller glia restore rod function in the $\mathrm{P} 23 \mathrm{H}$ rat. Stem Cells Transl Med. 2014;3(3):323-33. 
Fig. 1 Anatomical distribution of Müller glia within the retina

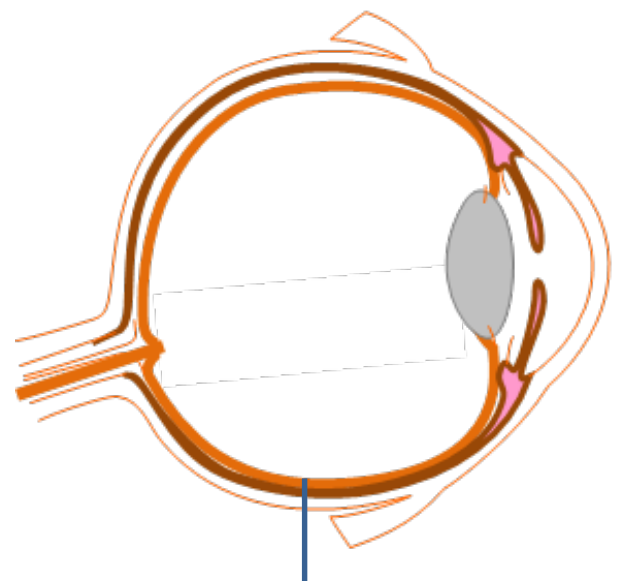

Neural retina

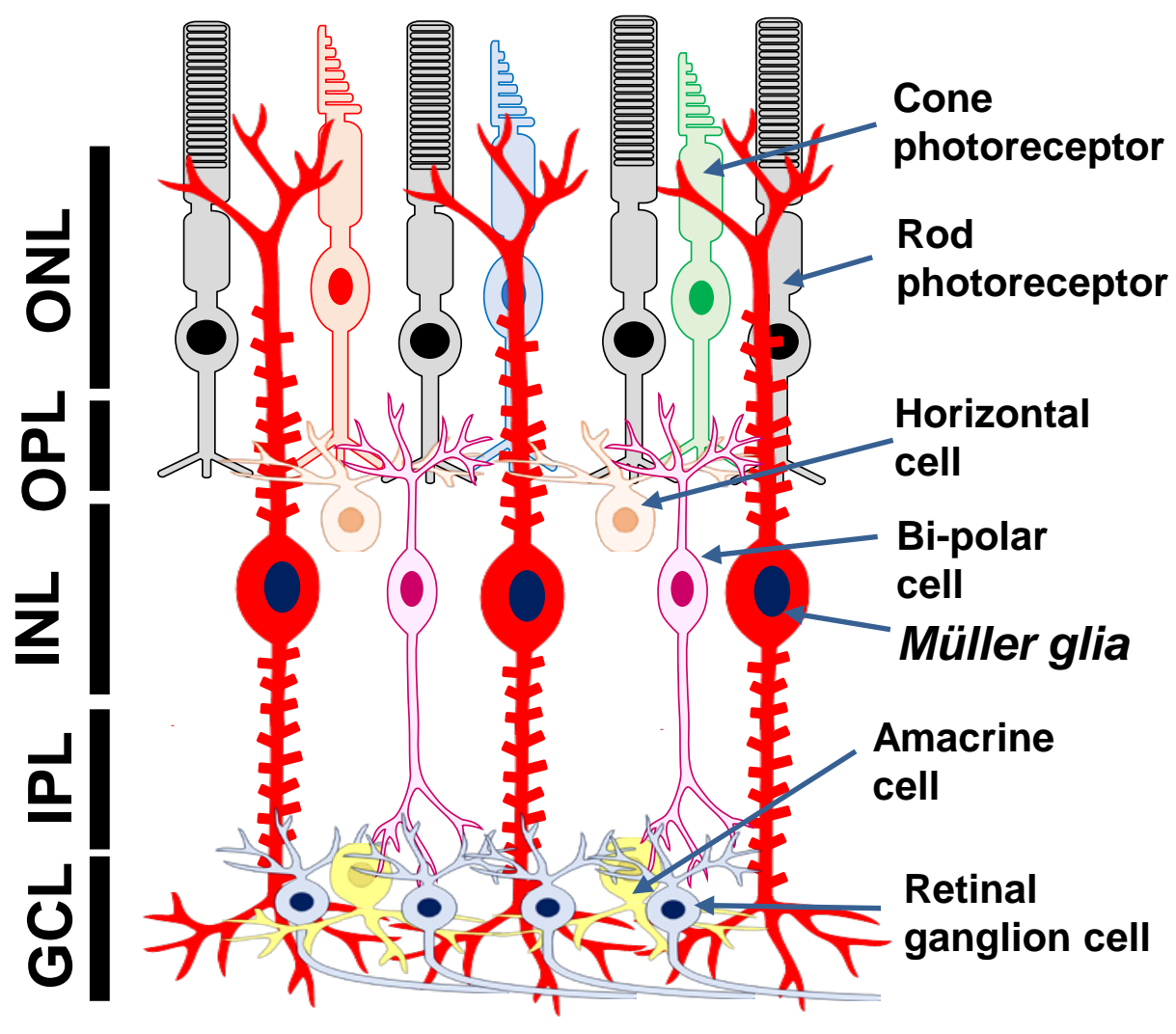


Fig. 2 Multiple neuroprotective functions of Müller glia

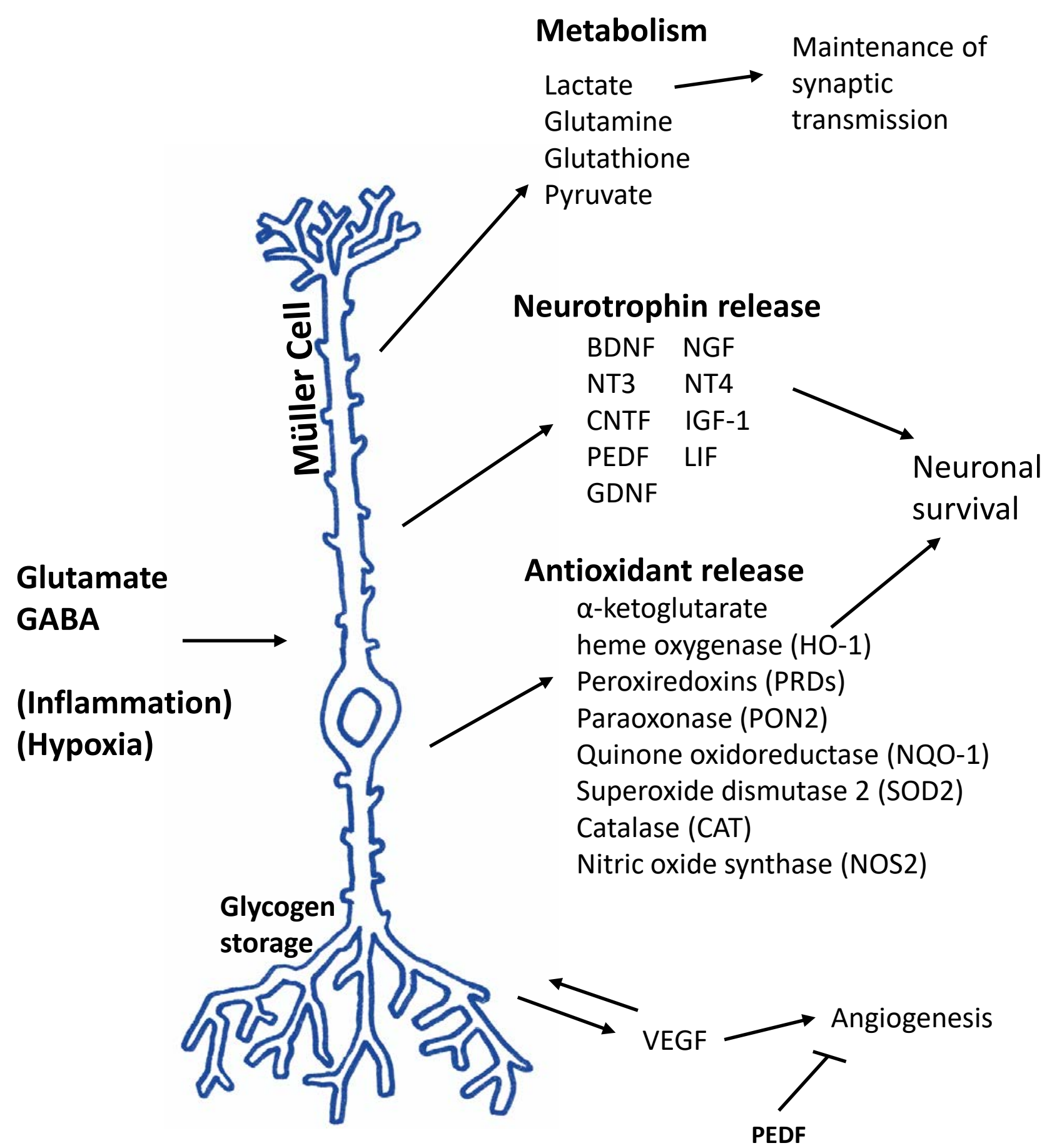

